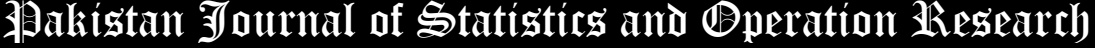

\section{Estimation of the Exponential Pareto Distribution's Parameters under Ranked and Double Ranked Set Sampling Designs}

\author{
Sabry M. A. H. ${ }^{1}$, Almetwally Ehab M. ${ }^{2, *}$
}

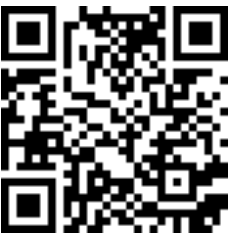

* Corresponding Author

1. Department of Mathematical Statistics, Faculty of Graduate Studies for Statistical Research, Cairo University, Cairo, Egypt. mohusss@cu.edu.eg

2. Faculty of Business Administration Delta University for Science and Technology. Egypt. ehabxp_2009@hotmail.com

\begin{abstract}
In this paper, the derivation of the likelihood function for parameter estimation based on double ranked set sampling (DRSS) designs used by Sabry et al. (2019) for the estimation of the parameters of the power generalized Weibull distribution is considered. The developed likelihood function is then used for the estimation of the exponential Pareto distribution parameters. The maximum likelihood estimators (MLEs) are then investigated and compared to the corresponding ones based on simple random sampling (SRS) and ranked set sampling (RSS) designs. A Monte Carlo simulation is conducted and the absolute relative biases, mean square errors, and efficiencies are compared for the different designs. The relative efficiency of the DRSS estimates with respect to other designs was found to be higher in case of the exponential Pareto distribution (EP).
\end{abstract}

Key Words: Simple Random Sampling; Ranked Set Sampling; Double Ranked Set Sampling; Estimation Parameter; Maximum Likelihood Estimation.

Mathematical Subject Classification: 60F10, 62D05

\section{Introduction}

McIntyre (1952), proposed the RSS design to help finding a more efficient estimate of the mean pasture yields. Takahasi and Wakimoto (1968) was the first who mathematically proved that RSS mean estimator is more efficient than its SRS competitor. RSS designs assume that there will be no errors in ranking the units with respect to the variable of interest. Al-Omari and Al-Hadhrami (2011) and Zamanzade and Mahdizadeh (2020) discussed this statement and found that for most practical applications there will imperfectly ranking and there will be a loss in efficiency of the estimators based on RSS. To reduce the errors in ranking, several modifications on the RSS procedure were made. For example, the extreme ranked set sampling (ERSS) design introduced by Samawi et al. (1996), the median ranked set sampling (MRSS) design introduced by Muttlak (1997, 2003), the moving extreme ranked set sampling (MERSS) design introduced by Al-Odat and Al-Saleh (2001), modification in ratio estimator using rank set sampling introduced by Al-Odat (2009) and the multistage ranked set sampling (MSRSS) introduced by Al-Saleh and Al-Omari (2002). Stratified pair ranked set sampling was also introduced by Mahdizadeh and Zamanzade (2018) and neurotic ranked set sampling by Zamanzade and Al-Omari (2016). For more examples see Dell and Clutter (1972), Al-Saleh and Al-Hadhrami (2003) Abu-Dayyeh and Al Sawi (2009), Abu-Dayyeh et al. (2013), Al-Omari et al. (2015), Zheng and Al-Saleh (2002).

According to Wolfe (2004), the procedure for obtaining the RSS samples can be summarized as follows: Step 1: Randomly select $\mathrm{m} 2$ units from a target population with cumulative distribution function (cdf) and probability 
density function (pdf) $F(x ; \theta)$.and $f(x ; \theta)$ respectively. Step 2: Allocate the $m^{2}$ selected units as randomly as possible into $\mathrm{m}$ sets, each of size $\mathrm{m}$. Step 3: Without yet knowing any values for the variable of interest, rank the units within each set with respect to variable of interest. This may be based on personal professional judgment or done with concomitant variable correlated with the variable of interest. Step 4: Choose a sample for actual quantification by including the smallest ranked unit in the first set, the second smallest ranked unit in the second set, the process is continues in this way until the largest ranked unit is selected from the last set. Step 5: Repeat steps 1 through 4 for c cycles to obtain a sample of size $n=m c$.

RSS uses only one observation, namely, $X_{(11) k}$ the lowest observation in the $k^{\text {th }}$ cycle, from this set, then $X_{(22) k}$ the second lowest from another independent set of $m$ observation, and finally $X_{(m m) k}$ the largest observation from a last set of $m$ observations. This process can be described in Figure 1 .

\begin{tabular}{ccccc|c}
\hline \multicolumn{5}{c|}{ unmeasured units } & $\begin{array}{c}\text { measured units } \\
\text { (RSS) }\end{array}$ \\
\hline$X_{(11) k}$ & $X_{(12) k}$ & $\ldots \ldots$ & $X_{(1(m-1)) k}$ & $X_{(1 m) k}$ & $\boldsymbol{X}_{(\mathbf{1 1}) k}$ \\
$X_{(21) k}$ & $X_{(22) k}$ & $\ldots \ldots$ & $X_{(2(m-1)) k}$ & $X_{(2 m) k}$ & $\boldsymbol{X}_{(\mathbf{2 2}) k}$ \\
$\vdots$ & $\vdots$ & & $\vdots$ & $\vdots$ & $\vdots$ \\
$X_{(m 1) k}$ & $X_{(m 2) k}$ & $\ldots .$. & $X_{(m(m-1)) k}$ & $X_{(m m) k}$ & $\boldsymbol{X}_{(\boldsymbol{m} \boldsymbol{m}) \boldsymbol{k}}$ \\
\hline
\end{tabular}

Figure 1. Display of $m^{2}$ observations in in the $k^{\text {th }}$ set cycle sets of size $m$

Let $\left\{X_{(i i) j}, i=1,2, \ldots, m ; k=1,2, \ldots, r\right\}$ be a ranked set sample where $m$, is the set size and $r$, is the number of cycles. In the rest of this paper and for simplification proposes we will use $X_{(i) j}$ instead of $X_{(i i) j}$ Then cdf and pdf of $X_{i ; j}$ are given by

$F_{i: m}\left(x_{(i) j} ; \boldsymbol{\theta}\right)=\sum_{t=i}^{m}\left(\begin{array}{c}m \\ t\end{array}\right)[F(x ; \boldsymbol{\theta})]^{t}[1-F(x ; \boldsymbol{\theta})]^{m-t}$

and

$f_{i: m}\left(x_{(i) j} ; \boldsymbol{\theta}\right)=\frac{m !}{(i-1) !(m-i) !} f(x ; \boldsymbol{\theta})[F(x ; \boldsymbol{\theta})]^{i-1}[1-F(x ; \boldsymbol{\theta})]^{m-i}$.

respectively, where, $-\infty<x_{(i) j}<\infty$. The joint pdf of $x_{(i) j}, i=1,2, \ldots, m, k=1,2, \ldots, c$ is then given by

$$
L\left(\boldsymbol{\theta} ; \boldsymbol{X}_{R}\right)=\prod_{k=1}^{c} \prod_{i=1}^{m} f_{i: m}(x ; \boldsymbol{\theta})
$$

In this paper, we introduce the likelihood function of the DRSS for estimation of the parameters of different lifetime distributions. Sections 2 and 3 discuss DRSS procedure and the joint probability of a DRSS sample. Section 4 introduces an estimation process for the exponential Pareto distribution parameters for different designs. Section 5 is devoted to simulation study for comparing the DRSS estimators with both SRS and RSS estimators. Results, conclusions and final remarks are presented in section 6.

\section{Double Ranked Set Sampling}

As mentioned by Al-Saleh and Al-Kadiri (2000), DRSS was found to be more efficient in estimating the population mean than both RSS and simple random sampling (SRS). Moreover and using the concept of "degree of distinguishability" between the sample observations, they showed that ranking in the second stage is easier than that in the first stage. According to Al-Omari and Jaber (2010), Mahdizadeh and Zamanzade (2017, 2019, 2020a,b) and Haq et al. (2013) The DRSS algorithm can be described as follows:

Step 1: Identify $m^{3}$ elements from a target population with cdf and pdf $F(x ; \theta)$.and $f(x ; \theta)$ respectively.

Step 2: Divide the $m^{3}$ elements randomly into $m$ sets each of size $m^{2}$ elements and use the usual RSS procedure on each set to obtain $m$ RSS each of size $m$ of the form

$$
\boldsymbol{X}_{R}=\left\{\left\{X_{(1) j}, X_{(2) j}, \ldots . X_{(m) j}\right\}, j=1,2, \ldots, m\right\}
$$

Step 3: Case I: For even set sizes $(m=2 r)$, select from the first $r$ sets the minimum ranked measurement and from the last $r$ sets select the maximum ranked measurement. 
The DRSS will be of the form

$X_{D(e)}=\left\{X_{1,1}, X_{1,2}, \ldots, X_{1, r}, X_{m,(r+1)}, X_{m,(r+2)}, \ldots, X_{m, m}\right\}$

where

$X_{1, j}=\min \left\{\left\{X_{(1) j}, X_{(2) j}, \ldots . X_{(m) j}\right\}, j=1,2, \ldots, r\right\}$,

and

$$
X_{m, k}=\max \left\{\left\{X_{(1) k}, X_{(2) k}, \ldots . X_{(m) k}\right\}, k=r+1, r+2, \ldots, m\right\}
$$

Case II: For odd set sizes $(m=2 r+1)$, select from the first $r$ sets the minimum ranked measurement, from the $(r+1)^{t h}$ set, select the median and from the last $r$ sets select the maximum measurement. The DRSS will be of the form

$X_{D(o)}=\left\{X_{1,1}, X_{1,2}, \ldots, X_{1, r}, X_{(r+1),(r+1)}, X_{m,(r+2)}, \ldots, X_{m, m}\right\}$

where $X_{(r+1),(r+1)}=\operatorname{median}\left\{\left\{X_{(1)(r+1)}, X_{(2)(r+1)}, \ldots . X_{(m)(r+1)}\right\}\right\}$.

\section{Joint Probability Distribution of DRSS}

In this section we will derive the joint probability distribution of a modified DRSS. It can be easily shown that for the minimum, maximum and median order statistics, the cdfs are given by $F_{1: n}(x ; \theta)=P_{1: n}(X \leq x)=1-$ $[1-F(x ; \theta)]^{n}, F_{n: n}(X ; \theta)=[F(x ; \theta)]^{n}$ and $F_{\text {med:n }}(x ; \theta)=\frac{(2 r+1) !}{(r) !(r) !} \sum_{w=0}^{r} \frac{(-1)^{w}\left(\begin{array}{c}r \\ w\end{array}\right)}{r+w+1}\left(F\left(x_{w} ; \theta\right)\right)^{r+w+1}$ for odd sample size $(n=2 r+1)$. For a DRSS with even set sizes $(m=2 r)$ defined in Eq. (3), the cdf and the pdf of $X_{1: m}^{j}, j=$ $1,2, \ldots, r$ are given by,

and

$$
F_{X_{1, j}}\left(x_{1, j} ; \theta\right)=P_{X_{1, j}}\left(X_{1, j} \leq x_{1, j}\right)=1-\left[1-F_{1: m}\left(x_{1, j} ; \theta\right)\right]^{m}
$$

$$
f_{X_{1, j}}\left(x_{1, j} ; \theta\right)=m f_{1: m}\left(x_{1, j} ; \theta\right)\left[1-F_{1: m}\left(x_{1, j} ; \theta\right)\right]^{m-1}
$$

respectively, and the likelihood function of the subsample $X_{1,1}, X_{1,2}, \ldots, X_{1, r}$ will be given by

$L_{1: m}(\theta)=\prod_{j=1}^{r}\left(m f_{1: m}\left(x_{1, j} ; \theta\right)\left[1-F_{1: m}\left(x_{1, j} ; \theta\right)\right]^{m-1}\right)$

Similarly the cdf and pdf of $X_{m: m}^{k}$ are given by

and

$$
F_{X_{m, k}}\left(x_{m, k} ; \theta\right)=P_{X_{m, k}}\left(X_{m, k} \leq x_{m, k}\right)=\left[F_{m: m}\left(x_{m, k} ; \theta\right)\right]^{m}
$$

$$
f_{X_{m, k}}\left(x_{m, k} ; \theta\right)=m f_{m: m}\left(x_{m, k} ; \theta\right)\left[F_{m: m}\left(x_{m, k} ; \theta\right)\right]^{m-1}
$$

respectively, and the joint pdf of the random vector $X_{m: m}^{r+1}, X_{m: m}^{r+2} \ldots, X_{m: m}^{m}$ is defined as

$L_{m: m}(\theta)=\prod_{k=r+1}^{m} m f_{m: m}\left(x_{m, k} ; \theta\right)\left[F_{m: m}\left(x_{m, k} ; \theta\right)\right]^{m-1}$

From Eqs. (5) and (6), the likelihood function for an even size DRSS will be of the form

$$
\begin{aligned}
& L\left(\theta ; X_{D(e)}\right)=\left[\prod_{j=1}^{r} f_{X_{1, j}}\left(x_{1, j} ; \theta\right)\right] \times\left[\prod_{k=r+1}^{m} f_{X_{m, k}}\left(x_{m, k} ; \theta\right)\right] . \\
& \quad=\left[\prod_{j=1}^{r} m f_{1: m}\left(x_{1, j} ; \theta\right)\left[1-F_{1: m}\left(x_{1, j} ; \theta\right)\right]^{m-1}\right],\left[\prod_{k=r+1}^{m}\left(m f_{m: m}\left(x_{m, k} ; \theta\right)\left[F_{m: m}\left(x_{m, k} ; \theta\right)\right]^{m-1}\right)\right] .(7)
\end{aligned}
$$

when $m$ is odd, say $m=2 r+1$, the cdf and pdf of $X_{(r+1),(r+1)}$ is given by

$$
F_{X_{(r+1),(r+1)}}\left(x_{(r+1),(r+1)} ; \theta\right)=\frac{(2 r+1) !}{(r) !(r) !} \sum_{l=0}^{r} \frac{(-1)^{l}\left(\begin{array}{l}
r \\
l
\end{array}\right)}{r+l+1}\left(F_{r+1: m}\left(x_{(r+1),(r+1)} ; \theta\right)\right)^{r+l+1}
$$

and

$$
\begin{aligned}
f_{X_{(r+1),(r+1)}}\left(x_{(r+1),(r+1)} ; \theta\right)= & \frac{(2 r+1) !}{(r) !(r) !} f_{r+1: m}\left(x_{(r+1),(r+1)} ; \theta\right) \\
& \times\left(F_{r+1: m}\left(x_{(r+1),(r+1)} ; \theta\right)\left(1-F_{r+1: m}\left(x_{(r+1),(r+1)} ; \theta\right)\right)\right)^{r}
\end{aligned}
$$

From Eqs. (5), (6) and (9), the likelihood function for an odd size DRSS will be of the form

$$
L_{X_{D(o)}}(\theta)=\left[\prod_{j=1}^{r} f_{X_{1, j}}\left(x_{1, j} ; \theta\right)\right] \times\left[\prod_{k=r+1}^{m} f_{X_{m, k}}\left(x_{m, k} ; \theta\right)\right]\left(f_{X_{(r+1),(r+1)}}\left(X_{(r+1),(r+1)} ; \theta\right)\right),
$$




$$
\begin{aligned}
= & {\left[\prod_{j=1}^{r} m f_{1: m}\left(x_{1, j} ; \theta\right)\left[1-F_{1: m}\left(x_{1, j} ; \theta\right)\right]^{m-1}\right] } \\
& \times\left[\prod_{k=r+1}^{m} m f_{m: m}\left(x_{m, k} ; \theta\right)\left[F_{m: m}\left(x_{m, k} ; \theta\right)\right]^{m-1}\right]\left[\frac{(2 r+1) !}{(r) !(r) !} f_{r+1: m}\left(x_{(r+1),(r+1)} ; \theta\right)\right. \\
& \left.\times\left(F_{r+1: m}\left(x_{(r+1),(r+1)} ; \theta\right)\left(1-F_{r+1: m}\left(x_{(r+1),(r+1)} ; \theta\right)\right)\right)^{r}\right]
\end{aligned}
$$

For more information about distributions of order statistics, the reader is recommended to David and Nagaraja (2005) and Barry (2008).

\section{Estimation of the Exponential Pareto Distribution Parameters}

The exponential Pareto distribution introduced by Al-Kadim and Boshi (2013), a new generalization of the Pareto distribution has been discussed by Almetwally and Ahmad (2020), Marshall-Olkin generalized Pareto distribution has been obtained by Ahmad and Almetwally (2020) and is assumed as an alternative to the most widely used lifetime distribution in reliability engineering, the Weibull (1951) distribution. It is a versatile distribution that can take on the characteristics of other types of distributions, based on the value of the shape parameter. The cdf, pdf, and the quantile functions of the exponential Pareto distribution are given by

$F(x ; \alpha, \lambda, \beta)=1-e^{-\lambda\left(\frac{x}{\alpha}\right)^{\beta}}$,

$f(x ; \alpha, \lambda, \beta)=\frac{\lambda \beta}{\alpha}\left(\frac{x}{\alpha}\right)^{\beta-1} e^{-\lambda\left(\frac{x}{\alpha}\right)^{\beta}}$,

and

$Q(u)=\alpha\left[\frac{-\ln (1-u)}{\lambda}\right]^{\frac{1}{\beta}}$,

respectively, where $x>0, \lambda>0, \beta>0, \alpha>0$ and $0<u<1$.

\subsection{Estimation Based on SRS}

Let $X_{1}, X_{2}, \ldots, X_{n}$ be independent and identically distributed random variables from exponential Pareto distribution with pdf given in Eq. (12). The likelihood function of $\lambda, \beta$ and $\alpha$ is given by

$$
L(\alpha, \lambda, \beta ; x)=\prod_{i=1}^{n} \frac{\lambda \beta}{\alpha}\left(\frac{x_{i}}{\alpha}\right)^{\beta-1} e^{-\lambda\left(\frac{x_{i}}{\alpha}\right)^{\beta}},
$$

and the log likelihood function is then derived as

$\ell(\alpha, \lambda, \beta)=n \log \lambda+n \log \beta-2 n \log \alpha+(\beta-1) \sum_{i=1}^{n} \log x_{i}-\sum_{i=1}^{n} \lambda\left(\frac{x_{i}}{\alpha}\right)^{\beta}$,

The MLEs of $\lambda, \beta$ and $\alpha$ are obtained by simultaneously solving the following normal equations:

$\frac{n}{\bar{\lambda}}-\sum_{i=1}^{n}\left(\frac{x_{i}}{\widehat{\alpha}}\right)^{\widehat{\beta}}=0$,

$\frac{n}{\bar{\beta}}+\sum_{i=1}^{n} \log x_{i}-\sum_{i=1}^{n} \hat{\lambda}\left(\frac{x_{i}}{\widehat{\alpha}}\right)^{\widehat{\beta}} \log x_{i}=0$,

and

$\frac{n}{\widehat{\alpha}}-\sum_{i=1}^{n} \hat{\lambda}\left(-\frac{x_{i}}{\widehat{\alpha}^{2}}\right)^{\widehat{\beta}}=0$,

Equations 14-16 don't have a closed form solution. Therefore, numerical techniques are used to solve for the MLEs of the scale and shape parameters.

\subsection{Estimation Based on RSS}

Let $\left\{X_{(i) j}, i=1,2, \ldots, m ; k=1,2, \ldots, c\right\}$ be a ranked set sample with cdf and pdf given in Eqs. (1) and (2), where $n$ is the set size, $c$ is the number of cycles and $n=m c$. The likelihood function of the RSS sample for exponential Pareto data is given by, 
$L_{r}(\alpha, \lambda, \beta ; x)=\prod_{j=1}^{r} \prod_{i=1}^{m} C_{i} f\left(x_{(i) k} ; \lambda, \beta\right)\left[F\left(x_{(i) k} ; \lambda, \beta\right)\right]^{i-1}\left[1-F\left(x_{(i) k} ; \lambda, \beta\right)\right]^{n-i}$,
$=\prod_{k=1}^{c} \prod_{i=1}^{m} C_{i}\left(\frac{\lambda \beta}{\alpha}\left(\frac{x_{(i) k}}{\alpha}\right)^{\beta-1} e^{-\lambda\left(\frac{x_{(i) k}}{\alpha}\right)^{\beta}}\right)\left(1-e^{-\lambda\left(\frac{x_{(i) k}}{\alpha}\right)^{\beta}}\right)^{i-1}\left(e^{-\lambda\left(\frac{x_{(i) k}}{\alpha}\right)^{\beta}}\right)^{m-i}$

where $C_{i}=\frac{n !}{(i-1) !(n-i) !}$. The log likelihood function is directly derived as follows

$$
\ell_{R S S}(\alpha, \lambda, \beta) \propto m c \log \lambda+m c \log \beta-m c \log \alpha+(\beta-1)\left(\sum_{k=1}^{c} \sum_{i=1}^{m} \log \left(\frac{x_{(i) k}}{\alpha}\right)\right)
$$

$-\lambda \sum_{k=1}^{c} \sum_{i=1}^{m}(m-i+1)\left(\frac{x_{(i) k}}{\alpha}\right)^{\beta}+\sum_{k=1}^{c} \sum_{i=1}^{m}(i-1) \log \left(1-e^{-\lambda\left(\frac{x_{(i) k}}{\alpha}\right)^{\beta}}\right)$,

and the likelihood normal equations become

$\frac{m c}{\widehat{\lambda}}-(m-i+1) \sum_{k=1}^{c} \sum_{i=1}^{m}\left(\frac{x_{(i) k}}{\widehat{\alpha}}\right)^{\widehat{\beta}}+\sum_{k=1}^{c} \sum_{i=1}^{m}(i-1) \frac{\left(\frac{x_{(i) k}}{\hat{\alpha}}\right)^{\widehat{\beta}} e^{-\widehat{\lambda}\left(\frac{x_{(i) k}}{\hat{\alpha}}\right)^{\widehat{\beta}}}}{1-e^{-\lambda\left(\frac{x_{(i) k}}{\hat{\alpha}}\right)^{\widehat{\beta}}}}=0$

$\frac{m c}{\widehat{\beta}}+\sum_{k=1}^{c} \sum_{i=1}^{m} \log \left(\frac{x_{(i) k}}{\widehat{\alpha}}\right)-\hat{\lambda}\left(\sum_{k=1}^{c} \sum_{i=1}^{m}(m-i+1)\left(\frac{x_{(i) k}}{\widehat{\alpha}}\right)^{\widehat{\beta}} \log \left(\frac{x_{(i) k}}{\widehat{\alpha}}\right)\right)$

$+\hat{\lambda}\left(\sum_{k=1}^{c} \sum_{i=1}^{m}(i-1) \frac{\left(\frac{x_{(i) k} \hat{\alpha}}{\hat{\beta}} e^{-\hat{\lambda}\left(\frac{x_{(i) k}}{\hat{\alpha}}\right)^{\widehat{\beta}}} \log \left(\frac{x_{(i) k}}{\hat{\alpha}}\right)\right.}{1-e^{-\hat{\lambda}\left(\frac{x_{(i) k}}{\hat{\alpha}}\right)^{\hat{\beta}}}}\right)=0$

and

$\frac{m c}{\widehat{\alpha}}+(\beta-1) \frac{m c}{\widehat{\alpha}}-\frac{\hat{\lambda} \widehat{\beta}}{\widehat{\alpha}}\left(\sum_{k=1}^{c} \sum_{i=1}^{m}(i-1) \frac{\left(\frac{x_{(i) k}}{\widehat{\alpha}}\right)^{\widehat{\beta}} e^{-\widehat{\lambda}\left(\frac{x_{(i) k}}{\hat{\alpha}}\right)^{\widehat{\beta}}}}{1-e^{-\lambda\left(\frac{x(i) k}{\hat{\alpha}}\right)^{\widehat{\beta}}}}\right)=0$

These three nonlinear equations can't be solved analytically and will be solved numerically.

\subsection{Estimation Based on DRSS}

According to Equations 7 and 10, the likelihood function for the exponential Pareto distribution is derived as follows.

Case I: $m$ even $(m=2 r)$

$L_{D(e)}(\theta)=\left[\prod_{j=1}^{r} m f_{1: m}\left(x_{1, j}\right)\left[1-F_{1: m}\left(x_{1, j}\right)\right]^{m-1}\right]\left[\prod_{k=r+1}^{m} m f_{m: m}\left(x_{m, k}\right)\left[F_{m: m}\left(x_{m, k}\right)\right]^{m-1}\right]$.

where

$f_{1: m}\left(x_{j}\right)=m f\left(x_{1, j}\right)\left[1-F\left(x_{1, j}\right)\right]^{m-1}, F_{1: m}\left(x_{1, j}\right)=1-\left[1-F\left(x_{1, j}\right)\right]^{m}$,

$f_{m: m}\left(x_{k}\right)=m f\left(x_{m, k}\right)\left[F\left(x_{m, k}\right)\right]^{m-1}$, and $F_{m: m}\left(x_{m, k}\right)=\left[F\left(x_{m, k}\right)\right]^{m}$.

The likelihood function is then given by

$$
\begin{aligned}
& L_{D(e)}(\theta)=\left[\prod_{j=1}^{r} m\left(m f\left(x_{1, j}\right)\left[1-F\left(x_{1, j}\right)\right]^{m-1}\right)\left[\left[1-F\left(x_{1, j}\right)\right]^{m}\right]^{m-1}\right] \\
& \times\left[\prod_{k=r+1}^{m} m\left(m f\left(x_{m, k}\right)\left[F\left(x_{m, k}\right)\right]^{m-1}\right)\left[\left[F\left(x_{m, k}\right)\right]^{m}\right]^{m-1}\right] . \\
& L_{D(e)}(\theta)=\left(\prod_{j=1}^{r} m\left[m \frac{\lambda \beta}{\alpha}\left(\frac{x_{1, j}}{\alpha}\right)^{\beta-1} e^{-\lambda\left(\frac{x_{1, j}}{\alpha}\right)^{\beta}}\left(e^{-\lambda\left(\frac{x_{1, j}}{\alpha}\right)^{\beta}}\right)^{m-1}\left(e^{-\lambda\left(\frac{x_{1, j}}{\alpha}\right)^{\beta}}\right)^{m(m-1)}\right]\right) \\
& \times\left(\prod_{k=r+1}^{m} m\left[m \frac{\lambda \beta}{\alpha}\left(\frac{x_{m, k}}{\alpha}\right)^{\beta-1} e^{-\lambda\left(\frac{x_{m, k}}{\alpha}\right)^{\beta}}\left(1-e^{-\lambda\left(\frac{x_{m, k}}{\alpha}\right)^{\beta}}\right)^{m-1}\left(1-e^{-\lambda\left(\frac{x_{m, k}}{\alpha}\right)^{\beta}}\right)^{m(m-1)}\right]\right) \\
& =\left(\prod_{j=1}^{r} m\left[m \frac{\lambda \beta}{\alpha}\left(\frac{x_{1, j}}{\alpha}\right)^{\beta-1} e^{-\lambda m^{2}\left(\frac{x_{1, j}}{\alpha}\right)^{\beta}}\right]\right)
\end{aligned}
$$




$$
\begin{aligned}
& \times\left(\prod_{k=r+1}^{m} m\left[\left(m \frac{\lambda \beta}{\alpha}\left(\frac{x_{m, k}}{\alpha}\right)^{\beta-1} e^{-\lambda\left(\frac{x_{m, k}}{\alpha}\right)^{\beta}}\right)\left(1-e^{-\lambda\left(\frac{x_{m, k}}{\alpha}\right)^{\beta}}\right)^{m^{2}-1}\right]\right) \\
& =m^{2 m} \lambda^{m} \beta^{m} \alpha^{-m-2 \beta+2}\left(\prod_{j=1}^{r} x_{1, j}^{\beta-1}\right)\left(\prod_{k=r+1}^{m} x_{m, k}^{\beta-1}\right)\left(e^{-\lambda m^{2} \sum_{j=1}^{r}\left(\frac{x_{1, j}}{\alpha}\right)^{\beta}}\right) \\
& \times\left(e^{-\lambda \sum_{k=r+1}^{m}\left(\frac{x_{m, k}}{\alpha}\right)^{\beta}}\right) \prod_{k=r+1}^{m}\left(1-e^{-\lambda\left(\frac{x_{m, k}}{\alpha}\right)^{\beta}}\right)^{m^{2}-1}
\end{aligned}
$$

Then, the associated log-likelihood function is obtained as

$$
\begin{aligned}
\ell_{D(e)}= & 2 m \log m+m \log \lambda+m \log \beta-(m-2 \beta+2) \log \alpha+(\beta-1)\left[\sum_{j=1}^{r} \log x_{1, j}+\sum_{k=r+1}^{m} \log x_{m, k}\right] \\
& -\lambda m^{2} \sum_{j=1}^{r}\left(\frac{x_{1, j}}{\alpha}\right)^{\beta}-\lambda \sum_{k=r+1}^{m}\left(\frac{x_{m, k}}{\alpha}\right)^{\beta}+\left(m^{2}-1\right) \sum_{k=r+1}^{m} \log \left(1-e^{-\lambda\left(\frac{x_{m, k}}{\alpha}\right)^{\beta}}\right) .
\end{aligned}
$$

The likelihood equations are therefore given by

$$
\begin{aligned}
& \frac{\partial \ell_{D(e)}}{\partial \lambda}=\frac{m}{\lambda}-m^{2} \sum_{j=1}^{r}\left(\frac{x_{1, j}}{\alpha}\right)^{\beta}-\sum_{k=r+1}^{m}\left(\frac{x_{m, k}}{\alpha}\right)^{\beta}+\left(m^{2}-1\right) \sum_{k=r+1}^{m} \frac{\left(\frac{x_{m, k}}{\alpha}\right)^{\beta} e^{-\lambda\left(\frac{x_{m, k}}{\alpha}\right)^{\beta}}}{1-e^{-\lambda\left(\frac{x_{m, k}}{\alpha}\right)^{\beta}}} \\
& \frac{\partial \ell_{D(e)}}{\partial \beta}=\frac{m}{\beta}+2 \log \alpha+\sum_{j=1}^{r} \log x_{1, j}+\sum_{k=r+1}^{m} \log x_{m, k}-\lambda m^{2} \sum_{j=1}^{r}\left(\frac{x_{1, j}}{\alpha}\right)^{\beta} \log \left(\frac{x_{1, j}}{\alpha}\right) \\
& -\lambda \sum_{k=r+1}^{m}\left(\frac{x_{m, k}}{\alpha}\right)^{\beta} \log \left(\frac{x_{m, k}}{\alpha}\right)+\left(m^{2}-1\right) \sum_{k=r+1}^{m} \frac{\lambda\left(\frac{x_{m, k}}{\alpha}\right)^{\beta} \log \left(\frac{x_{m, k}}{\alpha}\right) e^{-\lambda\left(\frac{x_{m, k}}{\alpha}\right)^{\beta}}}{1-e^{-\lambda\left(\frac{x_{m, k}}{\alpha}\right)^{\beta}}}
\end{aligned}
$$

and

$\frac{\partial \ell_{D(e)}}{\partial \alpha}=\frac{m-2 \beta+2}{\alpha}+\frac{\lambda \beta}{\alpha} m^{2} \sum_{j=1}^{r}\left(\frac{x_{1, j}}{\alpha}\right)^{\beta}-\frac{\lambda \beta}{\alpha} \sum_{k=\mathrm{r}+1}^{m}\left(\frac{x_{1, j}}{\alpha}\right)^{\beta}-\frac{\lambda \beta\left(m^{2}-1\right)}{\alpha} \sum_{k=r+1}^{m} \frac{\left(\frac{x_{m, k}}{\alpha}\right)^{\beta} e^{-\lambda\left(\frac{x_{m, k}}{\alpha}\right)^{\beta}}}{1-e^{-\lambda\left(\frac{x_{m, k}}{\alpha}\right)^{\beta}}}$

Case II: $m$ odd $(m=2 r+1)$

$$
\begin{aligned}
L_{D(o)}(\theta)= & {\left[\prod_{j=1}^{r} m f_{1: m}\left(x_{1, j}\right)\left[1-F_{1: m}\left(x_{1, j}\right)\right]^{m-1}\right]\left[\prod_{k=r+2}^{m} m f_{m: m}\left(x_{m, k}\right)\left[F_{m: m}\left(x_{m, k}\right)\right]^{m-1}\right] } \\
& \times\left[\frac{(2 r+1) !}{(r) !(r) !} f_{r+1: m}\left(x_{(r+1),(r+1)}\right)\left(F_{r+1: m}\left(x_{(r+1),(r+1)}\right)\left(1-F_{r+1: m}\left(x_{(r+1),(r+1)}\right)\right)\right)^{r}\right] \\
= & {\left[\prod_{j=1}^{r} m f_{1: m}\left(x_{1, j}\right)\left[1-F_{1: m}\left(x_{1, j}\right)\right]^{m-1}\right]\left[\prod_{k=r+2}^{m} m f_{m: m}\left(x_{m, k}\right)\left[F_{m: m}\left(x_{m, k}\right)\right]^{m-1}\right] } \\
& \times\left[\frac{(2 r+1) !}{(r) !(r) !}\left(\frac{m !}{r ! r !} f\left(x_{(r+1),(r+1)}\right)\left(F\left(x_{(r+1),(r+1)}\right)\right)^{r}\left(1-F\left(x_{(r+1),(r+1)}\right)\right)^{r}\right)\right] \\
& \times\left[F_{r+1: m}\left(x_{(r+1),(r+1)}\right)\left(1-F_{r+1: m}\left(x_{(r+1),(r+1)}\right)\right)\right]^{r}
\end{aligned}
$$

Since $F_{r+1: m}\left(x_{(r+1),(r+1)}\right)=\sum_{t=r+1}^{m}\left(\begin{array}{c}m \\ t\end{array}\right)\left(F\left(x_{(r+1),(r+1)}\right)\right)^{t}\left(1-F\left(x_{(r+1),(r+1)}\right)\right)^{m-t}$, then

$$
\begin{aligned}
& L_{D(o)}(\theta)=\left[\prod_{j=1}^{r} m\left(m \frac{\lambda \beta}{\alpha}\left(\frac{x_{1, j}}{\alpha}\right)^{\beta-1} e^{-\lambda m^{2}\left(\frac{x_{1, j}}{\alpha}\right)^{\beta}}\right)\right]\left(\prod _ { k = r + 2 } ^ { m } m \left[\left(m \frac{\lambda \beta}{\alpha}\left(\frac{x_{m, k}}{\alpha}\right)^{\beta-1} e^{-\lambda\left(\frac{x_{m, k}}{\alpha}\right)^{\beta}}\right)\right.\right. \\
& \left.\left.\times\left(1-e^{-\lambda\left(\frac{x_{m, k}}{\alpha}\right)^{\beta}}\right)^{m^{2}-1}\right]\right) \\
& \times\left[\frac { ( 2 r + 1 ) ! } { ( r ) ! ( r ) ! } \left(\frac{m !}{r ! r !}\left(\lambda \beta\left(\frac{x_{r+1, r+1}}{\alpha}\right)^{\beta-1} e^{-\lambda(r+1)\left(\frac{x_{r+1, r+1}}{\alpha}\right)^{\beta}}\left(1-e^{-\lambda\left(\frac{x_{r+1, r+1}}{\alpha}\right)^{\beta-1}}\right)^{r}\right)\right.\right. \\
& \times\left(F_{r+1: m}\left(x_{(r+1),(r+1)}\right)\right)^{r}\left(1-F_{r+1: m}\left(x_{(r+1),(r+1)}\right)\right)^{r} \\
& L_{D(o)}(\theta)=m^{2(m-1)} \lambda^{m} \beta^{m} \alpha^{-m-3 \beta+3}\left(\prod_{j=1}^{r} x_{1, j}^{\beta-1}\right)\left(\prod_{k=r+2}^{m} x_{m, k}^{\beta-1}\right)\left(e^{-\lambda m^{2} \sum_{j=1}^{r}\left(\frac{x_{1, j}}{\alpha}\right)^{\beta}}\right) \\
& \times\left(e^{-\lambda \sum_{k=r+2}^{m}\left(\frac{x_{m, k}}{\alpha}\right)^{\beta}}\right) \prod_{k=r+2}^{m}\left(1-e^{-\lambda\left(\frac{x_{m, k}}{\alpha}\right)^{\beta}}\right)^{\left(m^{2}-1\right)}
\end{aligned}
$$




$$
\begin{aligned}
& \times\left[\frac{(2 r+1) !}{(r) !(r) !}\left(\frac{m !}{r ! r !}\left(\frac{x_{r+1, r+1}}{\alpha}\right)^{\beta-1} e^{-\lambda(r+1)\left(\frac{x_{r+1, r+1}}{\alpha}\right)^{\beta-1}}\left(1-e^{-\lambda\left(\frac{x_{r+1, r+1}}{\alpha}\right)^{\beta-1}}\right)^{r}\right)\right] \\
& \times\left[\left(F_{r+1: m}\left(x_{(r+1),(r+1)}\right)\right)^{r}\left(1-F_{r+1: m}\left(x_{(r+1),(r+1)}\right)\right)^{r}\right]
\end{aligned}
$$

Then, the associated log-likelihood function is obtained as

$$
\begin{aligned}
\ell_{D(o)}= & 2(m-1) \log m+m \log \lambda+m \log \beta-(m+3 \beta-3) \log \alpha+(\beta-1) \sum_{j=1}^{r} \log x_{1, j} \\
& +(\beta-1) \sum_{k=r+2}^{m} \log x_{m, k}-\lambda m^{2} \sum_{j=1}^{r}\left(\frac{x_{1, j}}{\alpha}\right)^{\beta}-\lambda \sum_{k=r+2}^{m}\left(\frac{x_{m, k}}{\alpha}\right)^{\beta} \\
& +\left(m^{2}-1\right) \sum_{k=r+2}^{m} \log \left(1-e^{-\lambda\left(\frac{x_{m, k}}{\alpha}\right)^{\beta}}\right)+\log \left(\frac{(2 r+1) !}{(r) !(r) !} \times \frac{m !}{r ! r !}\right) \\
& +(\beta-1) \log \left(x_{(\mathrm{r}+1),(r+1)}\right)-\lambda(r+1)\left(\frac{x_{r+1, r+1}}{\alpha}\right)^{\beta}+r \log \left(1-e^{-\lambda\left(\frac{x_{r+1, r+1}}{\alpha}\right)^{\beta}}\right) \\
& +r\left[\log \left(F_{r+1: m}\left(x_{(r+1),(r+1)}\right)\right)+\log \left(1-F_{r+1: m}\left(x_{(r+1),(r+1)}\right)\right]\right.
\end{aligned}
$$

and the likelihood equations in this case are given by

$$
\begin{aligned}
& \frac{\partial \ell_{D(o)}}{\partial \lambda}=\frac{m}{\lambda}-m^{2} \sum_{j=1}^{r}\left(\frac{x_{1, j}}{\alpha}\right)^{\beta}-\sum_{k=r+2}^{m}\left(\frac{x_{m, k}}{\alpha}\right)^{\beta}+\left(m^{2}-1\right) \sum_{k=r+2}^{m}\left(\frac{\left(\frac{x_{m, k}}{\alpha}\right)^{\beta} e^{-\lambda\left(\frac{x_{m, k}}{\alpha}\right)^{\beta}}}{1-e^{-\lambda\left(\frac{x_{m, k}}{\alpha}\right)^{\beta}}}\right) \\
& -(r+1)\left(\frac{x_{r+1, r+1}}{\alpha}\right)^{\beta}+r\left(\frac{\left(\frac{x_{r+1, r+1}}{\alpha}\right)^{\beta} e^{-\lambda\left(\frac{x_{r+1, r+1}}{\alpha}\right)^{\beta}}}{1-e^{-\lambda\left(\frac{x_{r+1, r+1}}{\alpha}\right)^{\beta}}}\right)+F_{\lambda}\left(\frac{1-2 F_{r+1: m}\left(x_{(r+1),(r+1)}\right)}{F_{r+1: m}\left(x_{(r+1),(r+1)}\right)\left(1-F_{r+1: m}\left(x_{(r+1),(r+1)}\right)\right)}\right) \\
& \frac{\partial \ell_{D(o)}}{\partial \beta}=\frac{m}{\beta}-3 \log \alpha+\sum_{j=1}^{r} \log x_{1, j}+\sum_{k=r+2}^{m} \log x_{m, k}-\lambda m^{2} \sum_{j=1}^{r}\left(\frac{x_{1, j}}{\alpha}\right)^{\beta} \log \left(\frac{x_{1, j}}{\alpha}\right) \\
& -\lambda \sum_{k=r+2}^{m}\left(\frac{x_{m, k}}{\alpha}\right)^{\beta} \log \left(\frac{x_{m, k}}{\alpha}\right)+\left(m^{2}-1\right) \sum_{k=r+2}^{m} \frac{\lambda\left(\frac{x_{m, k}}{\alpha}\right)^{\beta} \log \left(\frac{x_{m, k}}{\alpha}\right) e^{-\lambda\left(\frac{x_{m, k}}{\alpha}\right)^{\beta}}}{1-e^{-\lambda\left(\frac{x_{m, k}}{\alpha}\right)^{\beta}}} \\
& +\log x_{(r+1),(r+1)}-\lambda(r+1)\left(\log \left(\frac{x_{r+1, r+1}}{\alpha}\right)\right)\left(\frac{x_{r+1, r+1}}{\alpha}\right)^{\beta} \\
& +r\left(\frac{\lambda\left(\frac{x_{r+1, r+1}}{\alpha}\right)^{\beta} \log \left(\frac{x_{r+1, r+1}}{\alpha}\right) e^{-\lambda\left(\frac{x_{r+1, r+1}}{\alpha}\right)^{\beta}}}{1-e^{-\lambda\left(\frac{x_{r+1, r+1}}{\alpha}\right)^{\beta}}}\right)+F_{\beta}\left(\frac{1-2 F_{r+1: m}\left(x_{(r+1),(r+1)}\right)}{F_{r+1: m}\left(x_{(r+1),(r+1)}\right)\left(1-F_{r+1: m}\left(x_{(r+1),(r+1))}\right)\right.}\right)
\end{aligned}
$$

and

$$
\begin{aligned}
& \frac{\partial \ell_{D(o)}}{\partial \alpha}=-\frac{m+3 \beta-3}{\alpha}+\frac{\lambda \beta m^{2}}{\alpha} \sum_{j=1}^{r}\left(\frac{x_{1, j}}{\alpha}\right)^{\beta}+\frac{\lambda \beta m^{2}}{\alpha} \sum_{k=\mathrm{r}+2}^{m}\left(\frac{x_{m, k}}{\alpha}\right)^{\beta}-\frac{\lambda \beta\left(m^{2}-1\right)}{\alpha} \sum_{k=r+2}^{m} \frac{\left(\frac{x_{m, k}}{\alpha}\right)^{\beta} e^{-\lambda\left(\frac{x_{m, k}}{\alpha}\right)^{\beta}}}{1-e^{-\lambda\left(\frac{x_{m, k}}{\alpha}\right)^{\beta}}}
\end{aligned}
$$

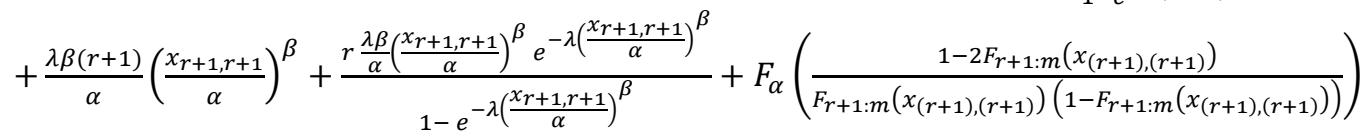

where

$F_{\lambda}=\partial F_{r+1: m}\left(x_{(r+1),(r+1)}\right) / \partial \lambda, F_{\beta}=\partial F_{r+1: m}\left(x_{(r+1),(r+1)}\right) / \partial \beta, F_{\alpha}=\partial F_{r+1: m}\left(x_{(r+1),(r+1)}\right) / \partial \alpha$.

Equating equation (23-25 odd sample sizes) or equations (27-29 even sample sizes) to zero and solving them simultaneously in both cases, maximum likelihoods estimators (MLEs) of $\lambda, \beta$ and $\alpha$ can be computed by solving the above likelihood equations. The above equations cannot give a closed form solution and for that, some numerical methods will be employed in order to obtain the desired estimates.

\section{Simulation Study}

In this section, we study a Monte Carlo simulation is conducted to compare the performance of the MLE methods under complete sample, ranked sample and double ranked sample designs. The data were generated from the exponential Pareto (EP) distribution for different values of $\alpha$ and $\beta$ while assuming $\lambda=1$ for simplicity reasons. The simulation algorithm is as follows: 


\section{For complete samples}

1) Generate $m$ random samples from the exponential Pareto distribution using the quantile function defined in Eq. (13) with 10000 replicates

2) For different sample sizes ( $m=6,9,10,15,20,25$ and 30), and different parameter values for $\alpha$ and $\beta$ are, $(\alpha=0.5,1.5$ and $3 ; \beta=0.5,1.5$ and 3$)$. Obtain the MLE.

3) Calculate the bias and mean square errors (MSE) of the estimates derived from Eqs. (14) and (15).

\section{For Ranked set samples}

1) Generate $n$ random samples from the exponential Pareto distribution using its quantile function with 10000 replicates.

2) Use the RSS method to simulate RSS samples as mentioned in section 1.

3) Repeat steps 1 and 2, $r$ (no. of cycles) times such that $m=n r$

4) Obtain the MLE by solving Eqs. (17) and (18) simultaneously and calculate the bias, MSE and relative efficiency of the RSS estimators compared to the SRS estimators where the relative efficiency of $\hat{\theta}_{2}$ compared with $\hat{\theta}_{1}$ is defined as

\section{For double ranked set samples}

$$
\operatorname{Eff}\left(\hat{\theta}_{1}, \hat{\theta}_{2}\right)=\frac{\operatorname{MSE}\left(\hat{\theta}_{1}\right)}{\operatorname{MSE}\left(\hat{\theta}_{2}\right)}
$$

1) Generate $m^{3}$ random samples from the Weibull distribution using the quantile function defined in Eq. (13) with 10000 replicates.

2) Use the DRSS method to simulate DRSS samples as mentioned in section 2 for even and odd sample sizes and obtain the MLE Eqs. (20-21) for odd samples and Eq. (23-24) for even samples.

3) Calculate the bias, MSE and relative efficiency of the DRSS estimators compared to the RSS estimators.

The results of the simulation study are reported in tables 1-6 and figures 1-3. From the tables it can be seen that

1) As the sample size increases the MSE decreases for both $\alpha$ and $\beta$ parameters.

2) For fixed $m$ and $\alpha$ as $\beta$ increases the MSE for both $\alpha$ and $\beta$ increases.

3) As $\alpha$ increases the MSE for both $\alpha$ and $\beta$ increases.

4) The relative efficiencies for both DRSS estimators are higher than 1, indicating better estimators compared to the both RSS and SRS estimators.

5) An over fitting problem may be an issue when using the proposed DRSS likelihood functions. 
Table 1: Bias, mean squared error (MSE) and efficiency of the estimators for $\alpha$ when $\alpha=0.5$ for all sampling

\begin{tabular}{|c|c|c|c|c|c|c|c|c|c|}
\hline \multirow{3}{*}{$m$} & \multirow{3}{*}{$\beta$} & \multicolumn{8}{|c|}{$\hat{\alpha}$} \\
\hline & & \multicolumn{2}{|c|}{ SRS } & \multicolumn{2}{|c|}{ RSS } & \multicolumn{2}{|c|}{ DRSS } & \multicolumn{2}{|c|}{ Efficiency } \\
\hline & & Bias & MSE & Bias & MSE & Bias & MSE & SRS/RSS & RSS/DRSS \\
\hline \multirow{3}{*}{6} & 0.5 & 0.1674 & 0.0333 & 0.0683 & 0.0152 & -0.0028 & 0.0085 & 2.1946 & 1.7838 \\
\hline & 1.5 & 0.2176 & 0.0499 & 0.0888 & 0.0179 & -0.0036 & 0.0099 & 2.7885 & 1.8081 \\
\hline & 3.0 & 0.2678 & 0.0599 & 0.1094 & 0.0315 & -0.0045 & 0.0159 & 1.9015 & 1.9811 \\
\hline \multirow{3}{*}{9} & 0.5 & 0.0924 & 0.0208 & 0.0413 & 0.0124 & -0.0947 & 0.0059 & 1.6727 & 2.1019 \\
\hline & 1.5 & 0.1664 & 0.0374 & 0.0619 & 0.0186 & -0.1515 & 0.0095 & 2.0072 & 1.9706 \\
\hline & 3.0 & 0.2995 & 0.0673 & 0.0929 & 0.0279 & -0.2424 & 0.0151 & 2.4087 & 1.8474 \\
\hline \multirow{3}{*}{10} & 0.5 & 0.0780 & 0.0104 & 0.0317 & 0.0053 & -0.0093 & 0.0028 & 1.9609 & 1.8915 \\
\hline & 1.5 & 0.1170 & 0.0135 & 0.0475 & 0.0079 & -0.0140 & 0.0049 & 1.6994 & 1.6213 \\
\hline & 3.0 & 0.1637 & 0.0156 & 0.0665 & 0.0111 & -0.0196 & 0.0056 & 1.4006 & 1.9861 \\
\hline \multirow{3}{*}{15} & 0.5 & 0.0486 & 0.0086 & 0.0148 & 0.0041 & -0.0087 & 0.0027 & 2.0879 & 1.5242 \\
\hline & 1.5 & 0.1020 & 0.0137 & 0.0267 & 0.0074 & -0.0139 & 0.0043 & 1.8559 & 1.7148 \\
\hline & 3.0 & 0.2143 & 0.0206 & 0.0481 & 0.0133 & -0.0222 & 0.0069 & 1.5466 & 1.9291 \\
\hline \multirow{3}{*}{20} & 0.5 & 0.0360 & 0.0051 & 0.0105 & 0.0022 & -0.0169 & 0.0012 & 2.3604 & 1.7935 \\
\hline & 1.5 & 0.0756 & 0.0081 & 0.0220 & 0.0045 & -0.0354 & 0.0032 & 1.7984 & 1.4124 \\
\hline & 3.0 & 0.1151 & 0.0107 & 0.0335 & 0.0099 & -0.0539 & 0.0081 & 1.0776 & 1.2222 \\
\hline \multirow{3}{*}{25} & 0.5 & 0.0283 & 0.0025 & 0.0089 & 0.0014 & -0.0081 & 0.0009 & 1.7509 & 1.6452 \\
\hline & 1.5 & 0.0537 & 0.0035 & 0.0160 & 0.0026 & -0.0105 & 0.0015 & 1.3618 & 1.7124 \\
\hline & 3.0 & 0.1020 & 0.0059 & 0.0288 & 0.0046 & -0.0136 & 0.0019 & 1.2862 & 2.4334 \\
\hline \multirow{3}{*}{30} & 0.5 & 0.0215 & 0.0023 & 0.0040 & 0.0010 & -0.0198 & 0.0006 & 2.4001 & 1.5094 \\
\hline & 1.5 & 0.0419 & 0.0035 & 0.0078 & 0.0019 & -0.0387 & 0.0010 & 1.8462 & 1.9153 \\
\hline & 3.0 & 0.0945 & 0.0049 & 0.0175 & 0.0043 & -0.0872 & 0.0022 & 1.1455 & 1.9866 \\
\hline
\end{tabular}

Table 2: Bias, mean squared error (MSE) and efficiency of the estimators for $\beta$ when $\alpha=0.5$ for all sampling designs.

\begin{tabular}{|c|c|c|c|c|c|c|c|c|c|}
\hline \multirow{3}{*}{$m$} & \multirow{3}{*}{$\beta$} & \multicolumn{8}{|c|}{$\hat{\beta}$} \\
\hline & & \multicolumn{2}{|c|}{ SRS } & \multicolumn{2}{|c|}{ RSS } & \multicolumn{2}{|c|}{ DRSS } & \multicolumn{2}{|c|}{ Efficiency } \\
\hline & & Bias & MSE & Bias & MSE & Bias & MSE & SRS/RSS & RSS/DRSS \\
\hline \multirow{3}{*}{6} & 0.5 & 0.1440 & 0.4171 & 0.0141 & 0.2132 & -0.0614 & 0.0896 & 1.9563 & 2.3793 \\
\hline & 1.5 & 0.3487 & 2.7577 & 0.0857 & 2.1643 & -0.4876 & 1.1728 & 1.2742 & 1.8455 \\
\hline & 3.0 & 0.8374 & 7.0006 & 0.1876 & 8.0245 & -0.7617 & 3.2899 & 1.6201 & 2.4392 \\
\hline \multirow{3}{*}{9} & 0.5 & 0.0970 & 0.1768 & 0.0180 & 0.1099 & 0.0304 & 0.0444 & 1.6086 & 2.4783 \\
\hline & 1.5 & 0.3258 & 1.7683 & 0.0356 & 0.8537 & 0.0932 & 0.3913 & 2.0715 & 2.1815 \\
\hline & 3.0 & 0.4624 & 6.3481 & 0.0595 & 3.3897 & 0.1689 & 1.5131 & 1.8727 & 2.2403 \\
\hline \multirow{3}{*}{10} & 0.5 & 0.0754 & 0.1602 & 0.0058 & 0.0794 & -0.0528 & 0.0374 & 2.0170 & 2.1262 \\
\hline & 1.5 & 0.2252 & 1.4242 & 0.0264 & 0.7450 & -0.3585 & 0.6990 & 1.9115 & 1.0659 \\
\hline & 3.0 & 0.3929 & 4.9415 & 0.0517 & 2.5802 & -0.7083 & 2.2336 & 1.9151 & 1.1552 \\
\hline \multirow{3}{*}{15} & 0.5 & 0.0397 & 0.0802 & 0.0049 & 0.0338 & 0.0228 & 0.0134 & 2.3717 & 2.5280 \\
\hline & 1.5 & 0.1292 & 0.8445 & 0.0081 & 0.3119 & 0.0604 & 0.1164 & 2.7079 & 2.6784 \\
\hline & 3.0 & 0.3018 & 3.3874 & 0.0267 & 1.2575 & 0.1334 & 0.4772 & 2.6938 & 2.6351 \\
\hline \multirow{3}{*}{20} & 0.5 & 0.0290 & 0.0665 & 0.0078 & 0.0214 & -0.0241 & 0.0037 & 3.1118 & 5.8318 \\
\hline & 1.5 & 0.1273 & 0.6224 & 0.0214 & 0.1858 & -0.1742 & 0.1215 & 3.3503 & 1.5286 \\
\hline & 3.0 & 0.2170 & 2.2980 & 0.0009 & 0.6732 & -0.0448 & 0.3555 & 3.4137 & 1.8935 \\
\hline \multirow{3}{*}{25} & 0.5 & 0.0283 & 0.0628 & -0.0022 & 0.0140 & 0.0154 & 0.0047 & 4.4935 & 2.9681 \\
\hline & 1.5 & 0.1549 & 0.6490 & 0.0138 & 0.1152 & 0.0464 & 0.0415 & 5.6357 & 2.7718 \\
\hline & 3.0 & 0.2629 & 2.3790 & -0.0294 & 0.4940 & 0.1030 & 0.2664 & 4.8155 & 1.8545 \\
\hline \multirow{3}{*}{30} & 0.5 & 0.0310 & 0.0430 & 0.0006 & 0.0099 & -0.0058 & 0.0064 & 4.3360 & 1.5406 \\
\hline & 1.5 & 0.0897 & 0.4256 & 0.0013 & 0.0825 & -0.0818 & 0.0332 & 5.1585 & 2.4856 \\
\hline & 3.0 & 0.1771 & 1.5732 & -0.0023 & 0.3222 & 0.0610 & 0.1959 & 4.8832 & 1.6444 \\
\hline
\end{tabular}


Table 3: Bias, mean squared error (MSE) and efficiency of the estimators for $\alpha$ when $\alpha=1.5$ for all sampling designs.

\begin{tabular}{|c|c|c|c|c|c|c|c|c|c|}
\hline \multirow{3}{*}{$\mathrm{m}$} & \multirow{3}{*}{$\beta$} & \multicolumn{8}{|c|}{$\hat{\alpha}$} \\
\hline & & \multicolumn{2}{|c|}{ SRS } & \multicolumn{2}{|c|}{ RSS } & \multicolumn{2}{|c|}{ DRSS } & \multicolumn{2}{|c|}{ Efficiency } \\
\hline & & Bias & MSE & Bias & MSE & Bias & MSE & SRS/RSS & RSS/DRSS \\
\hline \multirow{3}{*}{6} & 0.5 & 0.5022 & 1.0829 & 0.2050 & 0.2625 & -0.0045 & 0.1893 & 5.7193 & 1.3861 \\
\hline & 1.5 & 0.8211 & 1.1748 & 0.3352 & 0.4291 & -0.0074 & 0.3535 & 3.3229 & 1.2138 \\
\hline & 3.0 & 1.1049 & 1.2883 & 0.4511 & 0.5774 & -0.0099 & 0.4933 & 2.6116 & 1.1705 \\
\hline \multirow{3}{*}{9} & 0.5 & 0.2773 & 0.4124 & 0.1239 & 0.1192 & -0.2841 & 0.0902 & 4.5714 & 1.3211 \\
\hline & 1.5 & 0.4437 & 0.6598 & 0.2230 & 0.2145 & -0.5682 & 0.1804 & 3.6571 & 1.1890 \\
\hline & 3.0 & 0.7100 & 1.0557 & 0.4013 & 0.3861 & -1.1364 & 0.3608 & 2.9257 & 1.0701 \\
\hline \multirow{3}{*}{10} & 0.5 & 0.2339 & 0.1212 & 0.0950 & 0.1037 & -0.0271 & 0.0526 & 1.1687 & 1.9709 \\
\hline & 1.5 & 0.3509 & 0.1532 & 0.1425 & 0.1105 & -0.0406 & 0.0789 & 1.3864 & 1.4005 \\
\hline & 3.0 & 0.5263 & 0.2298 & 0.2138 & 0.1526 & -0.0609 & 0.1183 & 1.5059 & 1.2894 \\
\hline \multirow{3}{*}{15} & 0.5 & 0.1458 & 0.1545 & 0.0445 & 0.0444 & -0.0266 & 0.0239 & 3.4832 & 1.8521 \\
\hline & 1.5 & 0.2624 & 0.2781 & 0.0712 & 0.0710 & -0.0346 & 0.0311 & 3.9186 & 2.2795 \\
\hline & 3.0 & 0.4382 & 0.3568 & 0.1139 & 0.1135 & -0.0450 & 0.0405 & 3.1424 & 2.8056 \\
\hline \multirow{3}{*}{20} & 0.5 & 0.1079 & 0.1143 & 0.0314 & 0.0394 & -0.0505 & 0.0214 & 2.9033 & 1.8380 \\
\hline & 1.5 & 0.2051 & 0.2078 & 0.0596 & 0.0727 & -0.0959 & 0.0483 & 2.8583 & 1.5046 \\
\hline & 3.0 & 0.2699 & 0.2516 & 0.0785 & 0.0928 & -0.1262 & 0.0643 & 2.7112 & 1.4428 \\
\hline \multirow{3}{*}{25} & 0.5 & 0.0848 & 0.0750 & 0.0267 & 0.0284 & -0.0124 & 0.0190 & 2.6365 & 1.4957 \\
\hline & 1.5 & 0.1611 & 0.1424 & 0.0406 & 0.0432 & -0.0206 & 0.0315 & 3.2956 & 1.3697 \\
\hline & 3.0 & 0.3061 & 0.2706 & 0.0617 & 0.0657 & -0.0342 & 0.0524 & 4.1195 & 1.2544 \\
\hline \multirow{3}{*}{30} & 0.5 & 0.0644 & 0.0524 & 0.0119 & 0.0231 & -0.0101 & 0.0108 & 1.2741 & 1.9772 \\
\hline & 1.5 & 0.1159 & 0.1206 & 0.0215 & 0.0365 & -0.0181 & 0.0204 & 1.2262 & 1.8657 \\
\hline & 3.0 & 0.2087 & 0.2504 & 0.0387 & 0.0537 & -0.0246 & 0.0436 & 1.2449 & 1.6281 \\
\hline
\end{tabular}

Table 4: Bias, mean squared error (MSE) and efficiency of the estimators for $\beta$ when $\alpha=1.5$ for all sampling designs.

\begin{tabular}{|c|c|c|c|c|c|c|c|c|c|}
\hline \multirow{3}{*}{$\mathrm{m}$} & \multirow{3}{*}{$\beta$} & \multicolumn{8}{|c|}{$\hat{\beta}$} \\
\hline & & \multicolumn{2}{|c|}{ SRS } & \multicolumn{2}{|c|}{ RSS } & \multicolumn{2}{|c|}{ DRSS } & \multicolumn{2}{|c|}{ Efficiency } \\
\hline & & Bias & MSE & Bias & MSE & Bias & MSE & SRS/RSS & RSS/DRSS \\
\hline \multirow{3}{*}{6} & 0.5 & -0.0018 & 0.0201 & -0.0037 & 0.0115 & 0.0064 & 0.0048 & 1.7488 & 2.3941 \\
\hline & 1.5 & 0.0075 & 0.1909 & -0.0122 & 0.1050 & -0.0140 & 0.0416 & 1.8176 & 2.5221 \\
\hline & 3.0 & -0.0198 & 0.7386 & -0.0099 & 0.4476 & -0.0279 & 0.1665 & 1.6501 & 2.6875 \\
\hline \multirow{3}{*}{9} & 0.5 & -0.0028 & 0.0130 & -0.0009 & 0.0063 & 0.0062 & 0.0032 & 2.0755 & 1.9494 \\
\hline & 1.5 & -0.0234 & 0.1305 & -0.0067 & 0.0515 & 0.0215 & 0.0279 & 2.5327 & 1.8457 \\
\hline & 3.0 & -0.0094 & 0.4673 & 0.0058 & 0.2076 & 0.0475 & 0.1214 & 2.2504 & 1.7107 \\
\hline \multirow{3}{*}{10} & 0.5 & -0.0006 & 0.0126 & -0.0017 & 0.0045 & 0.0070 & 0.0058 & 2.8065 & 0.7694 \\
\hline & 1.5 & -0.0060 & 0.1142 & -0.0125 & 0.0427 & 0.0099 & 0.0200 & 2.6761 & 2.1293 \\
\hline & 3.0 & -0.0130 & 0.4097 & -0.0030 & 0.1737 & 0.0198 & 0.0940 & 2.3586 & 1.8476 \\
\hline \multirow{3}{*}{15} & 0.5 & 0.0011 & 0.0080 & -0.0008 & 0.0022 & 0.0077 & 0.0011 & 3.6714 & 1.9591 \\
\hline & 1.5 & -0.0078 & 0.0763 & -0.0041 & 0.0203 & 0.0214 & 0.0109 & 3.7524 & 1.8664 \\
\hline & 3.0 & -0.0111 & 0.3113 & -0.0085 & 0.0781 & 0.0419 & 0.0376 & 3.9858 & 2.0745 \\
\hline \multirow{3}{*}{20} & 0.5 & -0.0023 & 0.0059 & 0.0010 & 0.0012 & 0.0083 & 0.0011 & 4.8106 & 1.1691 \\
\hline & 1.5 & -0.0033 & 0.0464 & -0.0032 & 0.0107 & 0.0500 & 0.0096 & 4.3420 & 1.1176 \\
\hline & 3.0 & 0.0315 & 0.2025 & -0.0031 & 0.0479 & 0.0999 & 0.0382 & 4.2280 & 1.2536 \\
\hline \multirow{3}{*}{25} & 0.5 & -0.0011 & 0.0059 & -0.0012 & 0.0008 & 0.0055 & 0.0004 & 7.0495 & 2.3026 \\
\hline & 1.5 & -0.0074 & 0.0554 & -0.0011 & 0.0081 & 0.0158 & 0.0033 & 6.8690 & 2.4647 \\
\hline & 3.0 & 0.0110 & 0.2206 & -0.0040 & 0.0314 & 0.0351 & 0.0156 & 7.0283 & 2.0145 \\
\hline \multirow{3}{*}{30} & 0.5 & -0.0014 & 0.0032 & -0.0003 & 0.0007 & 0.0009 & 0.0003 & 4.3410 & 2.7631 \\
\hline & 1.5 & 0.0027 & 0.0329 & -0.0016 & 0.0083 & 0.0073 & 0.0048 & 3.9705 & 1.7203 \\
\hline & 3.0 & 0.0087 & 0.1012 & -0.0021 & 0.0309 & 0.0051 & 0.0159 & 3.2707 & 1.9491 \\
\hline
\end{tabular}


Table 5: Bias, mean squared error (MSE) and efficiency of the estimators for $\alpha$ when $\alpha=3$ for all sampling designs.

\begin{tabular}{|c|c|c|c|c|c|c|c|c|c|}
\hline \multirow{3}{*}{$\mathrm{m}$} & \multirow{3}{*}{$\beta$} & \\
\hline & & \multicolumn{2}{|c|}{ SRS } & \multicolumn{2}{|c|}{ RSS } & \multicolumn{2}{|c|}{ DRSS } & \multicolumn{2}{|c|}{ Efficiency } \\
\hline & & Bias & MSE & Bias & MSE & Bias & MSE & SRS/RSS & RSS/DRSS \\
\hline \multirow{3}{*}{6} & 0.5 & 0.7162 & 4.3698 & -1.4873 & 2.4211 & 0.0573 & 2.0013 & 1.8049 & 1.2097 \\
\hline & 1.5 & -0.0164 & 0.7323 & -0.6395 & 0.4664 & -0.0479 & 0.2012 & 1.5702 & 2.3179 \\
\hline & 3 & -0.0391 & 0.1851 & -0.3428 & 0.1359 & -0.0325 & 0.0514 & 1.3620 & 2.6437 \\
\hline \multirow{3}{*}{9} & 0.5 & 0.5280 & 3.5551 & -1.2867 & 1.7897 & 0.0420 & 1.3294 & 1.9865 & 1.3462 \\
\hline & 1.5 & -0.0078 & 0.5355 & -0.5228 & 0.3060 & -0.0297 & 0.1301 & 1.7500 & 2.3516 \\
\hline & 3 & -0.0265 & 0.1356 & -0.2759 & 0.0860 & -0.0203 & 0.0326 & 1.5757 & 2.6414 \\
\hline \multirow{3}{*}{10} & 0.5 & 0.4672 & 3.0955 & -1.1562 & 1.4303 & 0.0768 & 0.9444 & 2.1642 & 1.5146 \\
\hline & 1.5 & 0.0007 & 0.4523 & -0.4445 & 0.2164 & -0.0060 & 0.0939 & 2.0903 & 2.3057 \\
\hline & 3 & -0.0187 & 0.1143 & -0.2322 & 0.0594 & -0.0069 & 0.0234 & 1.9234 & 2.5453 \\
\hline \multirow{3}{*}{15} & 0.5 & 0.1668 & 2.9774 & -0.9736 & 0.9922 & 0.0255 & 0.4548 & 3.0009 & 2.1814 \\
\hline & 1.5 & -0.0397 & 0.2861 & -0.3652 & 0.1426 & -0.0077 & 0.0486 & 2.0060 & 2.9341 \\
\hline & 3 & -0.0187 & 0.1143 & -0.2322 & 0.0594 & -0.0069 & 0.0234 & 1.9234 & 2.5453 \\
\hline \multirow{3}{*}{20} & 0.5 & 0.2450 & 2.5244 & -0.7905 & 0.6504 & 0.0162 & 0.2034 & 3.8810 & 3.1983 \\
\hline & 1.5 & 0.0052 & 0.2261 & -0.2923 & 0.0896 & -0.0020 & 0.0222 & 2.5230 & 4.0331 \\
\hline & 3 & -0.0069 & 0.0568 & -0.1498 & 0.0236 & -0.0019 & 0.0056 & 2.4054 & 4.2496 \\
\hline & 0.5 & 0.1528 & 2.1846 & -0.7069 & 0.5169 & 0.0182 & 0.1430 & 4.2267 & 3.6156 \\
\hline \multirow[t]{2}{*}{25} & 1.5 & -0.0150 & 0.1945 & -0.2571 & 0.0692 & 0.0009 & 0.0155 & 2.8120 & 4.4682 \\
\hline & 3 & -0.0156 & 0.0485 & -0.1320 & 0.0182 & -0.0002 & 0.0039 & 2.6706 & 4.7057 \\
\hline \multirow{3}{*}{30} & 0.5 & 0.1479 & 1.4839 & -0.6173 & 0.3944 & 0.0006 & 0.0904 & 3.7628 & 4.3608 \\
\hline & 1.5 & 0.0014 & 0.1420 & -0.2236 & 0.0518 & -0.0031 & 0.0100 & 2.7430 & 5.1532 \\
\hline & 3 & -0.0052 & 0.0354 & -0.1129 & 0.0133 & -0.0020 & 0.0025 & 2.6709 & 5.2679 \\
\hline
\end{tabular}

Table 6: Bias, mean squared error (MSE) and efficiency of the estimators for $\beta$ when $\alpha=3$ for all sampling designs.

\begin{tabular}{|c|c|c|c|c|c|c|c|c|c|}
\hline \multirow{3}{*}{$\mathrm{m}$} & \multirow{3}{*}{$\beta$} & \multicolumn{8}{|c|}{$\hat{\beta}$} \\
\hline & & \multicolumn{2}{|c|}{ SRS } & \multicolumn{2}{|c|}{ RSS } & \multicolumn{2}{|c|}{ DRSS } & \multicolumn{2}{|c|}{ Efficiency } \\
\hline & & Bias & MSE & Bias & MSE & Bias & MSE & SRS/RSS & RSS/DRSS \\
\hline \multirow{3}{*}{6} & 0.5 & 0.1753 & 0.1350 & 0.0803 & 0.0337 & -0.0319 & 0.0062 & 4.0026 & 5.4438 \\
\hline & 1.5 & 0.5260 & 1.2149 & 0.2408 & 0.3035 & -0.0968 & 0.0558 & 4.0025 & 5.4381 \\
\hline & 3 & 1.0519 & 4.8596 & 0.4816 & 1.2141 & -0.1942 & 0.2233 & 4.0027 & 5.4362 \\
\hline \multirow{3}{*}{9} & 0.5 & 0.1062 & 0.0544 & 0.0506 & 0.0166 & -0.0498 & 0.0050 & 3.2743 & 3.3352 \\
\hline & 1.5 & 0.3187 & 0.4895 & 0.1518 & 0.1495 & -0.1491 & 0.0432 & 3.2744 & 3.4581 \\
\hline & 3 & 0.6374 & 1.9580 & 0.3035 & 0.5980 & -0.2984 & 0.1730 & 3.2742 & 3.4568 \\
\hline \multirow{3}{*}{10} & 0.5 & 0.0813 & 0.0403 & 0.0309 & 0.0085 & -0.0538 & 0.0042 & 4.7240 & 2.0364 \\
\hline & 1.5 & 0.2440 & 0.3628 & 0.0928 & 0.0768 & -0.1593 & 0.0371 & 4.7242 & 2.0715 \\
\hline & 3 & 0.4880 & 1.4512 & 0.1855 & 0.3072 & -0.3186 & 0.1483 & 4.7241 & 2.0712 \\
\hline \multirow{3}{*}{15} & 0.5 & 0.0580 & 0.0200 & 0.0158 & 0.0041 & -0.0547 & 0.0036 & 4.8866 & 1.1530 \\
\hline & 1.5 & 0.1740 & 0.1800 & 0.0475 & 0.0368 & -0.1600 & 0.0303 & 4.8868 & 1.2169 \\
\hline & 3 & 0.3480 & 0.7201 & 0.0950 & 0.1474 & -0.3200 & 0.1211 & 4.8868 & 1.2168 \\
\hline \multirow{3}{*}{20} & 0.5 & 0.0380 & 0.0117 & -0.0529 & 0.0030 & 0.0075 & 0.0020 & 3.8705 & 1.5091 \\
\hline & 1.5 & 0.1139 & 0.1049 & -0.1590 & 0.0272 & 0.0224 & 0.0180 & 3.8535 & 1.5154 \\
\hline & 3 & 0.2279 & 0.4196 & -0.3179 & 0.1085 & 0.0449 & 0.0718 & 3.8681 & 1.5098 \\
\hline \multirow{3}{*}{25} & 0.5 & 0.0334 & 0.0104 & -0.0526 & 0.0029 & 0.0063 & 0.0016 & 3.5885 & 1.8122 \\
\hline & 1.5 & 0.1003 & 0.0939 & -0.1572 & 0.0260 & 0.0188 & 0.0144 & 3.6169 & 1.7978 \\
\hline & 3 & 0.2006 & 0.3758 & -0.3076 & 0.0999 & 0.0375 & 0.0578 & 3.7624 & 1.7282 \\
\hline \multirow{3}{*}{30} & 0.5 & 0.0234 & 0.0072 & -0.0511 & 0.0027 & 0.0040 & 0.0009 & 2.6676 & 2.8441 \\
\hline & 1.5 & 0.0701 & 0.0647 & -0.1510 & 0.0236 & 0.0120 & 0.0085 & 2.7433 & 2.7658 \\
\hline & 3 & 0.1402 & 0.2587 & -0.3022 & 0.0946 & 0.0241 & 0.0341 & 2.7341 & 2.7749 \\
\hline
\end{tabular}



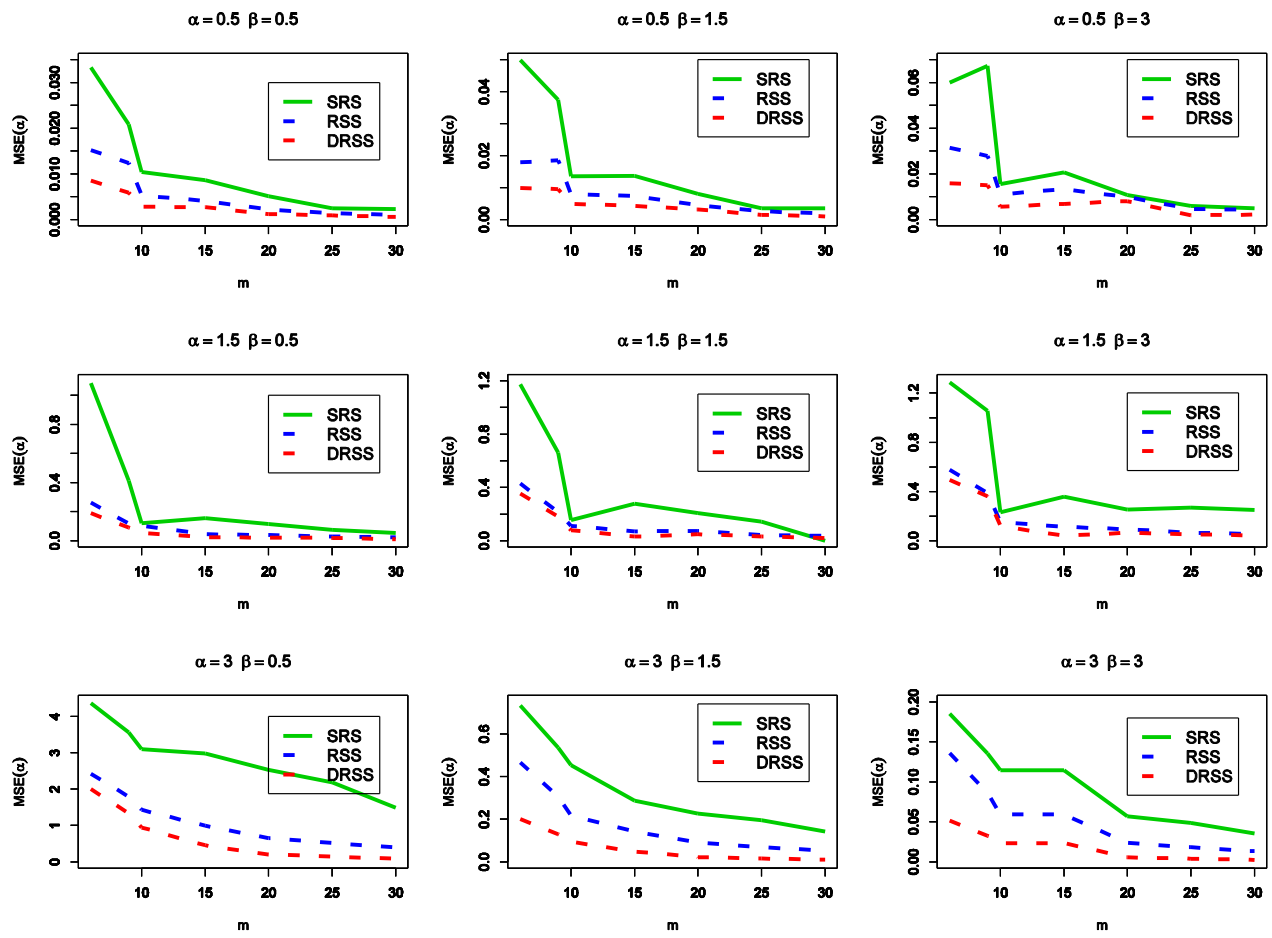

Figure 1: MSE of $\boldsymbol{\alpha}$ for different values for all sampling designs
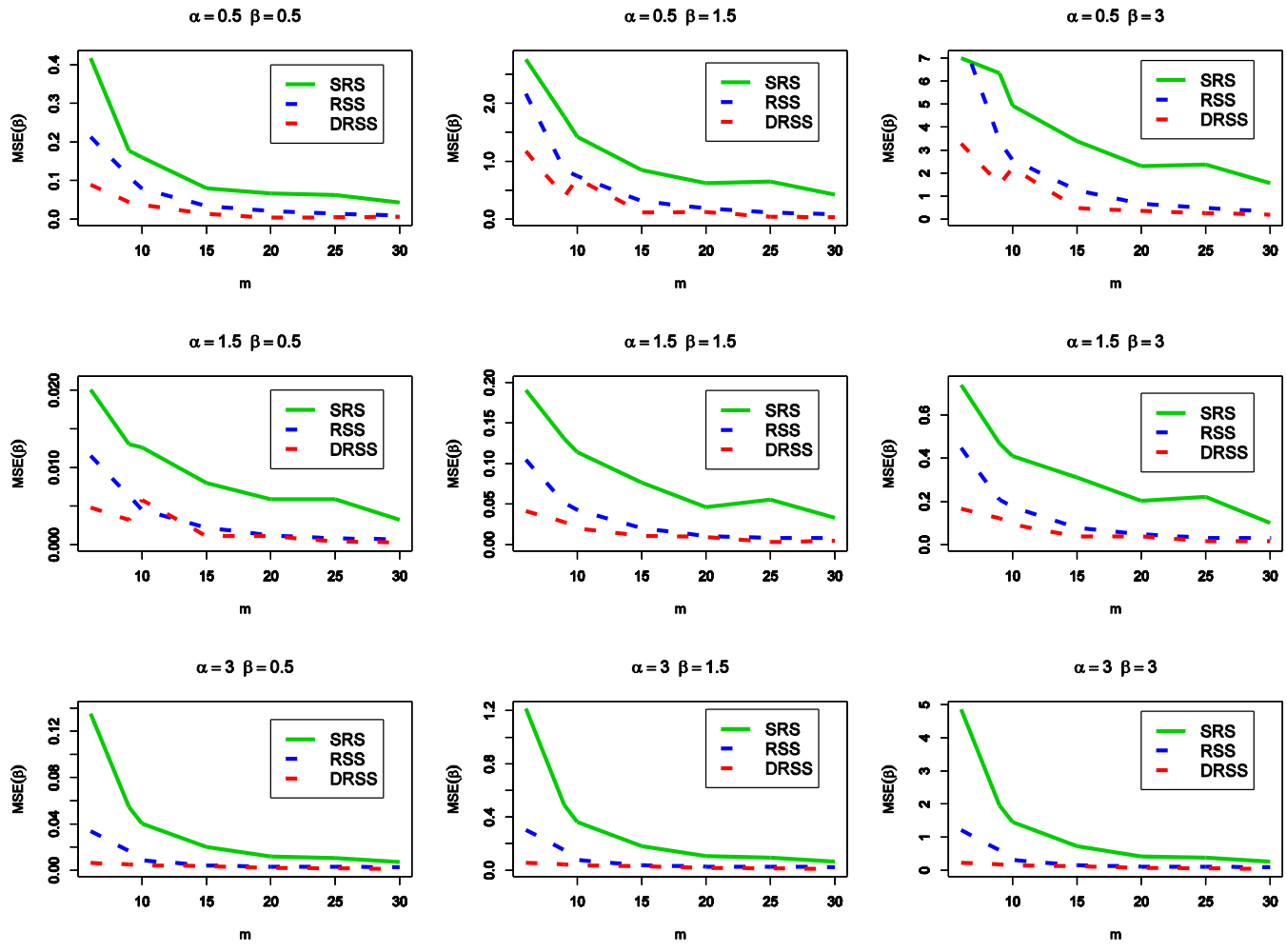

Figure 2: MSE of $\beta$ for different values for all sampling designs 


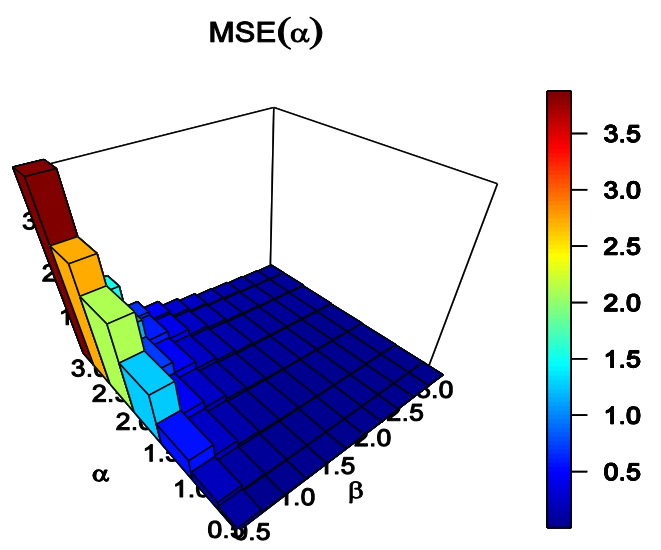

SRS

$\operatorname{MSE}(\alpha)$

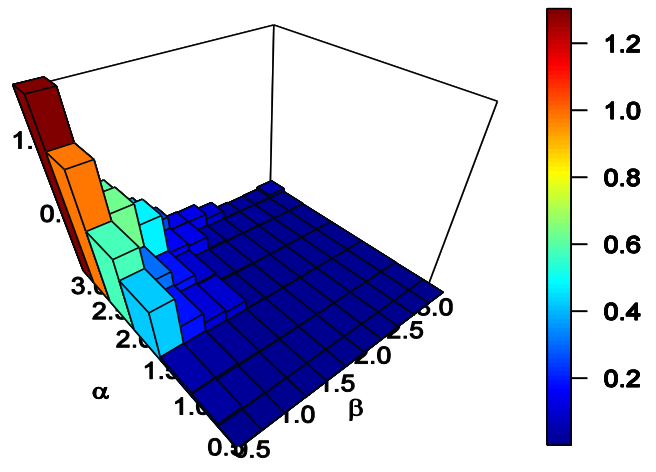

RSS

$\operatorname{MSE}(\alpha)$

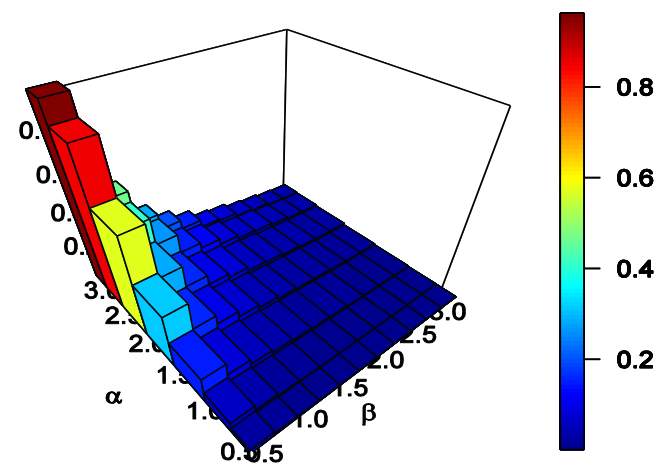

DRSS
$\operatorname{MSE}(\beta)$

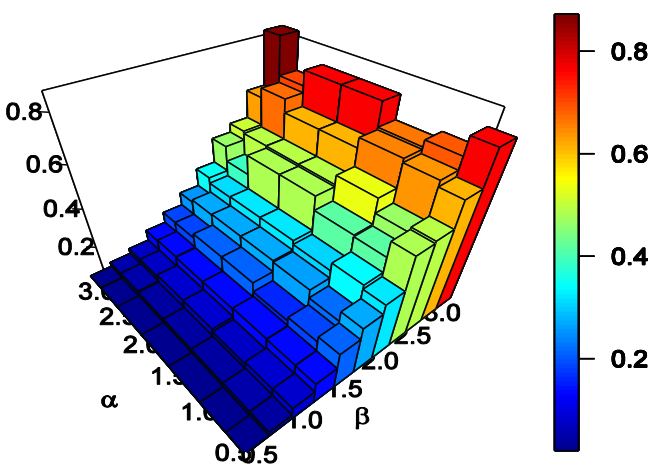

SRS

$\operatorname{MSE}(\beta)$

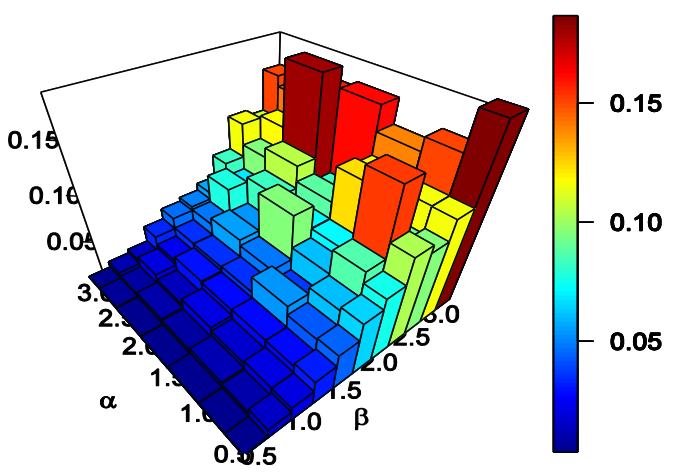

RSS

$\operatorname{MSE}(\beta)$

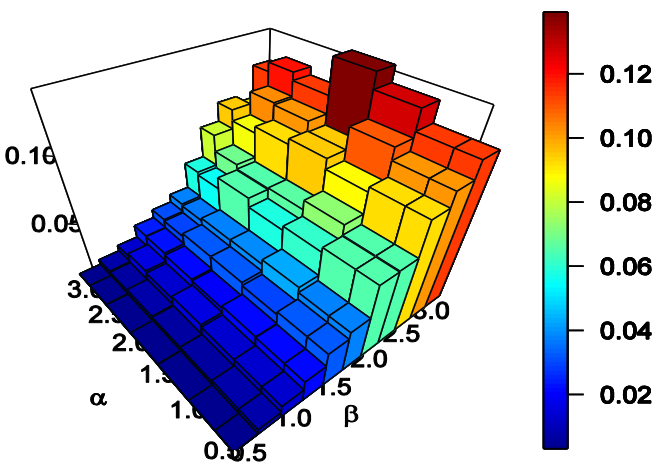

DRSS

Figure 3: 3-Dimention of MSE for different values for all sampling designs 


\section{Simulated Data Example}

In this section, we generate samples of the EP distribution as a simulated data by using this equation

$$
u_{i}=\left\{\begin{array}{ll}
\frac{i-\frac{3}{8}}{n+1-\frac{3}{4}}, & \text { if } n \leq 10 \\
\frac{i-\frac{1}{2}}{n}, & \text { if } n>10
\end{array}, i=1,2, \ldots, n,\right.
$$

From this generated data we select a SRS sample; a RSS sample and a DRSS sample each of size $n=3$ as follows SRS sample: we randomly select 3 numerical values from the generated data. RSS sample: we select 9 random values and assign them raw by raw after sorting in a matrix of size $3 \times 3$ and select the values on the diagonal. DRSS sample: First stage: we select 27 random values and assign them in 3 RSS matrices each of size $3 \times 3$. Second stage: from each matrix we select the minimum value from the values on the diagonal of matrix 1 , the median value from the values on of diagonal of matrix 2 and the maximum value from the values on the diagonal of matrix 3. The different samples are represented in table 7.

Table 7: Matrix of RSS and SRSS type of ranked sample

\begin{tabular}{c|c|c|c}
\hline & SRS & RSS & DRSS \\
\hline 1 & 0.5627 & 0.2081 & 0.3329 \\
\hline 2 & 1.2335 & 1.7077 & 1.1472 \\
\hline 3 & 2.1979 & 2.7055 & 1.9934
\end{tabular}

By comparing the fitted models based on the three different sampling designs, table 8 provides values of the estimated parameters for each sampling design and the corresponding standard errors (SE) along with Akaike information criterion (AIC), corrected AIC (CAIC) and Hannan-Quinn information criterion (HQIC) and Bayesian information criterion (BIC) for all fitted models.

Table 8: ML estimates, AIC, BIC, CAIC and HQIC for different Sample

\begin{tabular}{c|c|c|c|c|c|c}
\hline & \multicolumn{2}{|c|}{ SRS } & \multicolumn{2}{c|}{ RSS } & \multicolumn{2}{c}{ DRSS } \\
\hline & Estimate & SE & Estimate & SE & Estimate & SE \\
\hline alpha & 2.2011 & 666.6864 & 2.6029 & 466.5094 & 2.0074 & 291.7947 \\
\hline lambda & 2.2383 & 1015.5694 & 1.9094 & 729.0410 & 2.4151 & 706.2157 \\
\hline beta & 2.1408 & 1.0009 & 1.4907 & 0.7978 & 2.0116 & 0.6535 \\
\hline AIC & \multicolumn{2}{|c|}{11.7689} & 10.1893 & \multicolumn{2}{c}{7.3965} \\
\hline CAIC & \multicolumn{2}{|c|}{12.8123} & 11.2327 & \multicolumn{2}{c}{8.4400} \\
\hline HQIC & \multicolumn{2}{|c|}{12.9248} & \multicolumn{2}{c|}{11.3452} & \multicolumn{2}{c}{11.2840} \\
\hline BIC & \multicolumn{2}{|c|}{15.6564} & 14.0768 &
\end{tabular}

From table 8, it is clear that the model fitted under DRSS design is showing better results as compared to the other competitive models by providing smallest AIC, CAIC, HQIC and BIC which means that DRSS based models provide more accurate information about the true model used in this numerical example.

\section{Conclusion}

The likelihood function for DRSS designs were derived and maximum likelihood estimators for the exponential Pareto distribution were studied based on SRS, RSS and DRSS. It is clear from the simulation results and numerical example that the likelihood function used to estimate the parameters of the exponential Pareto distribution based on DRSS showed relatively efficient estimates compared to RSS estimators and the authors recommend using the DRSS designs and other two stage RSS designs as sampling designs when conducting parameter estimation of different probability distribution. For future work, the authors aim to generalize there work to other two-stage and multistage RSS designs. 


\section{Acknowledgment}

The authors are very grateful to the reviewers for their careful and fastidious perusing of the paper. The reviews are detailed and helpful to finalize the manuscript. The authors would like to kindly acknowledge them.

\section{References}

1. Abu-Dayyeh, W., \& Al Sawi, E. (2009). Modified inference about the mean of the exponential distribution using moving extreme ranked set sampling. Statistical Papers, 50(2), 249-259.

2. Abu-Dayyeh, W., Assrhani, A., \& Ibrahim, K. (2013). Estimation of the shape and scale parameters of Pareto distribution using ranked set sampling. Statistical Papers, 54(1), 207-225.

3. Al-Kadim, K. A., \& Boshi, M. A. (2013). exponential Pareto distribution. Mathematical Theory and Modeling, 3(5), 135-146.

4. Al-Odat, M. T., \& Al-Saleh, M. F. (2001). A variation of ranked set sampling. Journal of Applied Statistical Science, 10(2), 137-146.

5. Al-Odat, N. A. (2009). Modification in ratio estimator using rank set sampling. European Journal of Scientific Research, 29(2), 265-268.

6. Al-Omari, A. I., \& Al-Hadhrami, S. A. (2011). On maximum likelihood estimators of the parameters of a modified Weibull distribution using extreme ranked set sampling. Journal of Modern Applied Statistical Methods, 10(2), 18.

7. Al-Omari, A. I., \& Jaber, K. (2010). Improvement in estimating the population mean in double extreme ranked set sampling. In International Mathematical Forum (Vol. 5, No. 26, pp. 1265-1275).

8. Al-Omari, A. I., Al-Zubi, L. M., \& Khazaleh, A. (2015). On the population median estimation using quartile double ranked set sampling. Pakistan Journal of Statistics and Operation Research, 11(4), 513524.

9. Al-Saleh, M. F., \& Al-Hadhrami, S. A. (2003). Estimation of the mean of the exponential distribution using moving extremes ranked set sampling. Statistical Papers, 44(3), 367-382.

10. Al-Saleh, M. F., \& Al-Kadiri, M. A. (2000). Double-ranked set sampling. Statistics \& Probability Letters, 48(2), 205-212.

11. Al-Saleh, M. F., \& Al-Omari, A. I. (2002). Multistage ranked set sampling. Journal of Statistical planning and Inference, 102(2), 273-286.

12. Almetwally, E. M., \& Ahmad, H. A. H. (2020). A new generalization of the Pareto distribution and its applications. Statistics in Transition New Series, 21(5), 61-84.

13. Ahmad, H. H., \& Almetwally, E. (2020). Marshall-Olkin generalized Pareto distribution: Bayesian and non Bayesian estimation. Pakistan Journal of Statistics and Operation Research, 16(1), 21-33.

14. Barry C. A., Balakrishnan N., Nagaraja, H. N., (2008)." A first course in Order Statistics". Classics in Applied Mathematics, Siam, Society for Industrial and Applied Mathematics- Philadelphia.

15. David H. A., Nagaraja H. N., (2005). "Order Statistics". Wiley Series in Probability and Statistics, John Wiley \& Sons, Inc.

16. Dell, T. R., \& Clutter, J. L. (1972). Ranked set sampling theory with order statistics background. Biometrics, 28, 545-555.

17. Haq, A., Brown, J., Moltchanova, E., \& Al-Omari, A. I. (2013). Partial ranked set sampling design. Environmetrics, 24(3), 201-207.

18. Mahdizadeh, M. and Zamanzade, E. (2018). Stratified pair ranked set sampling. Communications in Statistics-Theory and Methods, 47(24), 5904-5915.

19. Mahdizadeh, M. and Zamanzade, E. (2020b). Smooth estimation of the area under the ROC curve in multistage ranked set sampling, to appear in Statistical Papers. DOI: https://doi.org/10.1007/s00362019-01151-6.

20. Mahdizadeh, M., \& Zamanzade, E. (2017). Reliability estimation in multistage ranked set sampling. REVSTAT: A Statistical Journal, 15(4), 565-581.

21. Mahdizadeh, M., \& Zamanzade, E. (2019). Efficient body fat estimation using multistage pair ranked set sampling. Statistical methods in medical research, 28(1), 223-234.

22. Mahdizadeh, M., \& Zamanzade, E. (2020a). Estimation of a symmetric distribution function in multistage ranked set sampling. Statistical Papers, 61(2), 851-867.

23. McIntyre, G. A. (1952). A method for unbiased selective sampling, using ranked sets. Australian journal of agricultural research, 3(4), 385-390.

24. Muttlak, H. A. (1997). Median ranked set sampling. Journal of Applied Statistics Sciences, 6, 245-255. 
25. Muttlak, H. A. (2003). Modified ranked set sampling methods. Pakistan Journal of Statistics, 19(3), 315-324.

26. Sabry, M. A., Muhammed, H. Z., Nabih, A., \& Shaaban, M. (2019). Parameter estimation for the power generalized Weibull distribution based on one-and two-stage ranked set sampling designs. J Stat Appl Probab, 8(2019), 113-128.

27. Samawi, H. M., Ahmed, M. S., \& Abu-Dayyeh, W. (1996). Estimating the population mean using extreme ranked set sampling. Biometrical Journal, 38(5), 577-586.

28. Takahasi, K., \& Wakimoto, K. (1968). On unbiased estimates of the population mean based on the sample stratified by means of ordering. Annals of the institute of statistical mathematics, 20(1), 1-31.

29. Weibull, W. (1951). A statistical distribution function of wide applicability. Journal of applied mechanics, 18(3), 293-297.

30. Wolfe, D. A. (2004). Ranked set sampling: an approach to more efficient data collection. Statistical Science, 19(4), 636-643.

31. Zamanzade, E., \& Al-Omari, A. I. (2016). New ranked set sampling for estimating the population mean and variance. Hacettepe Journal of Mathematics and Statistics, 45(6), 1891-1905.

32. Zamanzade, E., \& Mahdizadeh, M. (2020). Using ranked set sampling with extreme ranks in estimating the population proportion. Statistical methods in medical research, 29(1), 165-177.

33. Zheng, G., \& Al-Saleh, M. F. (2002). Modified maximum likelihood estimators based on ranked set samples. Annals of the Institute of Statistical Mathematics, 54(3), 641-658. 